Collection SFN 11 (2010) 71-88

(C) Owned by the authors, published by EDP Sciences, 2010

DOI: $10.1051 / \mathrm{sfn} / 201011005$

\title{
La Diffusion de Neutrons aux Petits Angles : mécanique statistique des liquides et traitement des données
}

\author{
L. Belloni
}

\author{
CEAISACLAY, SIS2M, Laboratoire Interdisciplinaire sur l'Organisation Nanométrique et \\ Supramoléculaire, 91191 Gif-sur-Yvette Cedex, France
}

\begin{abstract}
Small angle scattering is well suited for the study of so-called colloidal solutions which contain nanometric particles immersed in a more or less continuous and complex solvent. The goal is to extract from the scattering spectrum (scattering angle dependence of the scattering intensity) individual informations about the colloids (size, shape ... ) and collective ones (local arrangement, colloidal interaction). The present chapter will focus on the latter and will show how robust and powerful models of statistical mechanics of liquids are able to relate the scattering intensity to the microscopic pair potential of interaction between spherical particles and to allow intensive treatment of experimental data. More ambitious studies which involve the details of the supporting medium (discreteness of the ions or of the solvent molecules) or the presence of anisotropic particles (rods, plates ... ) requires heavier treatments which will be briefly surveyed at the end of the chapter.
\end{abstract}

Résumé. La diffusion de rayonnement aux petits angles est bien adaptée à l'étude des solutions dites colloïdales qui contiennent des particules de taille nanométrique plongées dans un solvant plus ou moins continu et complexe. Il s'agit d'extraire du spectre de diffusion (intensité diffusée fonction de l'angle de diffusion) des informations individuelles sur les colloïdes (taille, forme, ... ) et des informations collectives (arrangement local des particules les unes autour des autres, interaction colloïdale). Le chapitre présent va mettre l'accent sur ce second type d'information et montrer comment des modèles pratiques et performants de mécanique statistique des liquides sont capables de relier facilement l'intensité diffusée au potentiel d'interaction microscopique entre particules sphériques et sont adaptés au traitement intensif de données expérimentales. L'étude plus ambitieuse des détails du fluide porteur (description explicite des ions, voire des molécules de solvant) ou de particules anisotropes (bâtonnets, plaquettes ... ) requiert des traitements plus lourds qui seront évoqués en fin de chapitre.

\section{INTRODUCTION}

Le but du jeu de la diffusion de neutron aux petits angles (DNPA) et plus généralement de la diffusion de rayonnement est d'apporter des informations sur ce qui se passe au niveau microscopique au sein de la solution sous le faisceau. La gamme standard de vecteur de diffusion $q$, disons de l'ordre de $10^{-2}-1 \AA^{-1}$, est tout à fait adaptée à l'étude de solution colloïdales contenant des particules de taille nanométrique, les colloïdes, plongées dans un solvant, généralement l'eau, qui peut être enrichi d'ions ou de petites molécules (petits polymères, sucres ....). Un chapitre précédent est dédié à cette physique dite de la matière molle. Vue l'échelle d'observation, il est naturel et tentant d'ignorer formellement les détails du fluide porteur et de considérer explicitement uniquement les collö̈des plongés dans un continuum. L'intensité diffusée n'est due essentiellement qu'à ces grosses particules, le solvant n'intervenant que via la notion de contraste. Les nombreuses questions auxquelles la diffusion de rayonnement peut apporter une réponse sont généralement de deux types. Le premier concerne les propriétés individuelles des

This is an Open Access article distributed under the terms of the Creative Commons Attribution-Noncommercial License 3.0, which permits unrestricted use, distribution, and reproduction in any noncommercial medium, provided the original work is properly cited. 
particules: Quelle est la taille $R$ de la protéine? Quel est le nombre d'agrégation de la micelle? Sont-elles sphériques ou anisotropes? Les tubes d'imogolite ont-ils une ou deux parois? Y-a-t-il une polydispersité de la solution? La seconde catégorie de questions s'intéresse au contraire aux propriétés collectives du fluide colloïdal: comment les particules interagissent deux à deux? Quel est l'interaction ou la force colloïdale? Comment cette interaction de paires induit-elle un arrangement local des particules les unes autour des autres au sein d'une solution à concentration donnée? J'ai écrit "au contraire" car les deux types d'information visés requièrent des expériences et des réflexes différents. Pour les propriétés individuelles, on veut pouvoir observer les particules indépendamment les unes des autres, on dilue donc beaucoup quitte à perdre du signal, on ajoute du sel pour éteindre les interactions électrostatiques éventuelles, on maudit les corrélations ou interférences restantes entre particules qui polluent le facteur de forme recherché, on trace l'intensité diffusée $I(q)$ en échelle log-log afin de magnifier le comportement aux grands $q(>1 / R)$ qui donnent les détails des particules ... Au contraire, si on s'intéresse au couplage entre particules, on concentre, on déionise, on trace $I(q)$ en échelle linéaire, on assume une forme individuelle simple, et on se focalise sur le facteur de structure à petit $q(<1 / R)$ en regrettant que le facteur de forme décroissant tue de l'information intéressante trop rapidement. Le chapitre présent va plutôt se placer dans cette seconde optique. En clair, la question principale sera: j'ai mesuré $I(q)$ de ma solution à concentration colloïdale, composition du solvant et températures données, comment puis-je remonter au potentiel de paires $v(r)$ (énergie d'interaction en joules fonction de la séparation $r$ ) ou à la force de paires $(-\mathrm{d} v / \mathrm{d} r$ en newtons) entre deux colloïdes? Contrainte supplémentaire, j'ai effectué cette expérience de diffusion sur une centaine d'échantillons, je voudrais donc pouvoir effectuer cette analyse de données de manière efficace, précise mais rapide. Il faut donc mettre à contribution les techniques modernes de mécanique statistique des liquides qui, justement, sont centrées autour de cette même question. On montrera en particulier comment les modèles basés sur les équations intégrales, approchées mais précises, permettent l'analyse intensive de spectres expérimentaux dans le cas de particules colloïdales sphériques. Les études plus ambitieuses qui s'intéressent aux détails du fluide porteur, aux ions ou, pourquoi pas, aux molécules d'eau, ou qui ont affaire à des particules anisotropes, demandent un traitement théorique et numérique plus difficile, moins adapté à l'analyse de routine. Néanmoins, on donnera quelques exemples de ce qui peut être fait en pratique et de ce qui ne peut pas. Tout ce qui a été dit dans cette introduction et tout ce qui va être développé dans la suite est très général à la diffusion de rayonnement (lumière, $\mathrm{X}$, neutrons). Dans la mesure du possible, on donnera des exemples spécifiques de DNPA.

\section{INTENSITÉ DIFFUSÉE, FACTEUR DE FORME, FACTEUR DE STRUCTURE}

L'intensité diffusée s'écrit comme le module au carré de l'amplitude (champ électrique pour faire simple) diffusée. Elle va donc être gouvernée par les interférences entre ondes diffusées par les différents éléments (les noyaux en DNPA) de la solution :

$$
I(q)=\left\langle\sum_{m} \sum_{n} b_{m} b_{n} e^{i \vec{q}\left(\vec{r}_{m}-\vec{r}_{n}\right)}\right\rangle
$$

où le vecteur $r_{m}$ pointe la position absolue du noyau $m, b_{m}$ représente sa longueur de diffusion relative (par rapport au solvant de même volume). $I(q)$ de l'équation 1 est donc la section efficace de la solution, qu'il faut ensuite diviser par le volume diffusant pour une normalisation complète. Les deux crochets \langle\rangle indiquent qu'il faut faire la moyenne statistique de cette somme d'interférences, moyenne sur les conformations, les orientations, les répartitions spatiales, ces moyennes étant en général couplées. A l'aide de la figure schématique 1, il est pratique de repérer la position relative $s_{m}$ d'un noyau par rapport à la position absolue $R_{i}$ du colloïde $i$ auquel il appartient. Les vecteurs s'ajoutent dans l'espace réel, les 


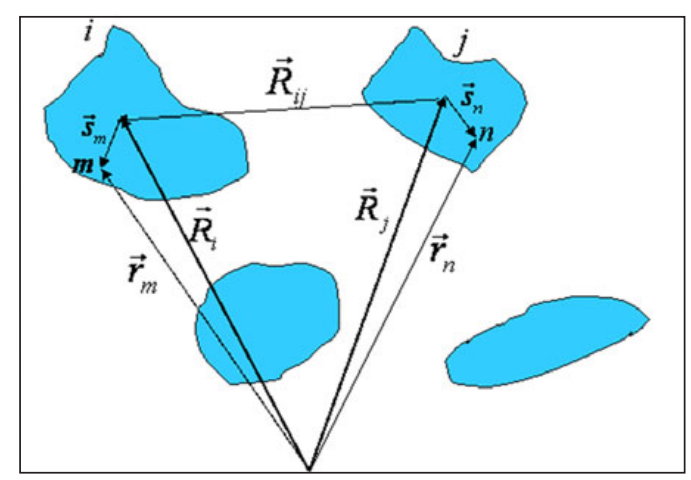

Figure 1. Solution colloïdale. Positions absolues et relatives des éléments diffusants, positions absolues des colloïdes.

facteurs se multiplient dans l'espace de Fourier :

$$
\begin{aligned}
& I(q)=\left\langle\sum_{i, j}\left(\sum_{m \in i} b_{m} e^{i \vec{q} \vec{s}_{m}}\right)\left(\sum_{n \in j} b_{n} e^{-i \vec{q} \vec{s}_{n}}\right) e^{i \vec{q} \vec{R}_{i j}}\right\rangle \\
& I(q)=\left\langle\sum_{i, j} f_{i}(\vec{q}) f_{j}^{*}(\vec{q}) e^{i \vec{q} \vec{R}_{i j}}\right\rangle \equiv \sum_{i}\left\langle\left|f_{i}(\vec{q})\right|^{2}\right\rangle+\left\langle\sum_{i \neq j} f_{i}(\vec{q}) f_{j}^{*}(\vec{q}) e^{i \vec{q} \vec{R}_{i j}}\right\rangle .
\end{aligned}
$$

Grouper les amplitudes dues à tous les éléments diffusants d'un même colloïde $i$ fait apparaître son facteur d'amplitude ("intra") $f_{i}(q)$ (à une conformation et une orientation par rapport à $q$ données). Les corrélations/interférences entre particules différentes ("inter”) se retrouvent dans le facteur de phase $e^{i \vec{q} \vec{R}_{i j}}=e^{i \vec{q}\left(\vec{R}_{i}-\vec{R}_{j}\right)}$. Il faut ensuite faire la moyenne du produit en réalisant que les facteurs intra et inter sont généralement couplés (une protéine peut se déformer au contact d'une voisine donc $f_{i}$ dépend de $R_{i j}$, des bâtonnets s'approchent différemment suivant leur orientation relative donc $R_{i j}$ dépend de $f_{i} \ldots$... Tout ce qu'on a pu faire dans (2) est d'isoler les contributions "self", $i=j$, et faire apparaître ce qu'on va appeler le facteur de forme d'une particule. Le reste, $i \neq j$, ne disparaît qu'en l'absence de couplage entre colloïdes, soit en solution diluée, soit en solution idéale.

\subsection{Particules indépendantes - Solution diluée - Facteur de forme}

Dans ce cas limite, le dernier terme dans (2) s'annule, les intensités dues à chacune des particules s'ajoutent sans déphasage et, pour $N$ particules identiques :

$$
I(q)=\sum_{i} P_{i}(q)=N P(q)
$$

avec différentes expressions équivalentes pour le facteur de forme $P(q)$, moyenne du module au carré du facteur d'amplitude, somme des interférences entre ondes diffusées par l'ensemble des noyaux (ou électrons) d'un colloïde:

$$
\begin{aligned}
& P(q)=\left\langle\left|\sum_{m} b_{m} e^{i \vec{q} \vec{q}_{m}}\right|^{2}\right\rangle=\left\langle\sum_{m, n} b_{m} b_{n} e^{i \vec{q} \vec{q}_{m n}}\right\rangle=\left\langle\sum_{m, n} b_{m} b_{n} \frac{\sin q s_{m n}}{q s_{m n}}\right\rangle \\
& P(q)=\left\langle\left|\int_{\text {particule }} \Delta \rho(\vec{s}) e^{i \vec{q} \vec{s}} d \vec{s}\right|^{2}\right\rangle=\left\langle\iint_{\text {particule }} \Delta \rho\left(\vec{s}^{\prime}\right) \Delta \rho\left(\vec{s}+\vec{s}^{\prime}\right) e^{i \vec{q} \vec{s}} d \vec{s}^{\prime} d \vec{s}\right\rangle .
\end{aligned}
$$


La $1^{\text {ière }}$ ligne utilise la notation discrète, somme sur tous les sites diffusants. En présence de centaines ou de milliers d'atomes, il est important d'apprécier en pratique la différence numérique entre la 1 ère expression qui ne fait intervenir qu'une somme simple mais qui requiert d'effectuer une moyenne angulaire sur l'orientation relative entre la particule et le vecteur $q$ et la dernière expression qui a déjà effectué cette moyenne analytiquement (d'où les $\sin q s_{m n} / q s_{m n}$ ) mais au prix de faire apparaître une somme double et des racines carrées (pour avoir les distance $r_{m n}$ ), rapidement prohibitives en temps de calcul! La seconde ligne utilise plutôt une représentation intégrale de la densité (relative) de longueur de diffusion $\Delta \rho(s)$. Etant donnée la résolution limite de l'expérience, il est inutile de rentrer dans des détails de taille inférieure à $1 / q_{\max }$ et on peut traiter un ensemble d'atomes voisins ou de molécules voisines comme un petit volume de densité uniforme.

Des expressions analytiques très populaires existent pour de nombreuses formes simples, à commencer bien-sûr par le cas d'une sphère homogène de rayon $R$ (volume $V_{R}$ ) et densité uniforme $\Delta \rho$ :

$$
P(q)=\left(\Delta \rho V_{R}\right)^{2}\left(\frac{\sin q R-q R \cos q R}{(q R)^{3} / 3}\right)^{2} .
$$

L'analyse du spectre en solution idéale consiste dans ce cas à essayer différentes tailles $R$ et à comparer intensités expérimentale et théorique en log-log. Pratiquement tout le temps, les belles oscillations (avec zéros parfaits) présentes dans la courbe théorique n'apparaissent que "lissées" dans les données expérimentales. Si la résolution instrumentale ne suffit pas pour expliquer cet écart, il est alors tentant d'invoquer une certaine polydispersité de taille de sphères. Pour une répartition gaussienne (en nombre) de rayons sphériques, centrée autour de $R_{0}$ et d'écart type $\sigma$, une expression analytique tout autant pratique et néanmoins moins connue est ( $N$ dans l'équation 3 représente alors le nombre total de sphères):

$$
P(q)=\frac{8 \pi^{2} \Delta \rho^{2}}{q^{6}}\left[1+q^{2}\left(R_{0}^{2}+\sigma^{2}\right)-\left\{\begin{array}{l}
\left(1-q^{2}\left(R_{0}^{2}-3 \sigma^{2}\right)+4 q^{4} \sigma^{4}\right) \cos 2 q R_{0} \\
+2 q R_{0}\left(1+2 q^{2} \sigma^{2}\right) \sin 2 q R_{0}
\end{array}\right\} e^{-2 q^{2} \sigma^{2}}\right] .
$$

Au-delà de la sphère pleine, on peut essayer des sphères stratifiées, des ellipsoïdes, des cylindres, des formes à oreilles de Mickey (molécules colloïdales), des chaînes vermiculaires ...Si la forme globale des colloïdes est connue ou suspectée a priori, il suffit d'ajuster un ou quelques paramètres (taille, allongement ...). Parfois, cette recherche primaire est insuffisante et il faut avoir un peu plus d'imagination, comme dans le cas des tubes d'imogolite à double paroi déjà évoqués [1].

On peut aussi être plus ambitieux et essayer d'extraire la structure du colloïde $a b$ initio, sans aucune hypothèse a priori sur la répartition spatiale des atomes qui le composent. On espère ainsi obtenir la structure interne "basse résolution" d'une protéine, par opposition à la structure "haute résolution" obtenue par diffraction d'un cristal. La procédure est itérative: on remplit un volume (celui que va occuper in fine le colloïde construit) à l'aide de petites cases correspondant à des sites, des résidus, des groupes d'atomes ..., de positions, tailles et longueurs de diffusion ajustables. De nouveau, la taille des cases n'a pas besoin de descendre en dessous de $1 / q_{\max }$. On calcule le facteur de forme correspondant à l'aide de l'une des expressions (4). Enfin, on cherche à minimiser l'écart entre spectres théorique et expérimental en ajustant les nombreux paramètres tout en respectant certaines contraintes de connectivité, compacité ... (on a quand même une vague idée de ses particules, si ce n'est de la chimie!). La connaissance simultanée de plusieurs spectres obtenus à des contrastes différents (mélanges de solvant en DNPA) est bien-sûr bienvenue. La minimisation qui peut faire intervenir des centaines de paramètres est l'étape critique et fait appel à des techniques modernes de simulation Monte Carlo avec recuit simulé qu'il n'est pas possible de détailler ici. Il suffit de dire que cette méthode moderne a obtenu des succès certains et est capable d'obtenir une structure très précise de particules comme le 70s Escherichia coli Ribosome [2]. Dans certains cas, la structure extraite de la diffusion aux petits angles a même été obtenue avant que la synthèse de cristal n'ait pu être réalisée et a été confirmée seulement plus tard par la diffraction! 


\subsection{Particules corrélées en interaction - Solution concentrée de sphères - Facteur de structure}

On revient à l'expression générale (2) de l'intensité :

$$
I(q)=\left\langle\sum_{i, j} f_{i} f_{j}^{*} e^{i \vec{q} \vec{R}_{i j}}\right\rangle .
$$

Cette fois-ci, les positions, orientations, conformations des différentes particules sont corrélées et il faut donc savoir traiter $10^{23}$ particules en même temps! On va utiliser pour cela tout l'arsenal de mécanique statistique des liquides développé depuis les années 1960 et appliqué en pratique aux solutions colloïdales et à la diffusion de rayonnement depuis les années 1980. On va considérer maintenant que les particules sont sphériques et rigides (et toutes identiques pour l'instant, juste pour simplifier les notations). Dans ce cas, et dans ce cas seulement j'insiste, les facteurs d'amplitude et de forme sont indépendants de l'orientation et de la position des particules (si l'on a eu la présence d'esprit de choisir l'origine des particules en leur centre!) et les moyennes intra et inter sont découplées :

$$
I(q)=P(q)\left\langle\sum_{i, j} e^{i \vec{q} \vec{R}_{i j}}\right\rangle=N P(q) S(q)
$$

où apparaît le facteur de structure $S(q)$ qui est la signature (pour ne pas dire la transformée de Fourier, $\mathrm{TF})$ des positions relatives des $N$ particules au sein de la solution :

$$
\begin{aligned}
S(q) & =\frac{1}{N}\left\langle\sum_{i=1}^{N} \sum_{j=1}^{N} e^{i \vec{q} \vec{R}_{i j}}\right\rangle=1+(N-1)\left\langle e^{i \vec{q} \overleftarrow{R}_{12}}\right\rangle \\
& =1+\rho \int_{\text {volumeV }} g(r) e^{i \vec{q} \vec{r}} d \vec{r}=1+\rho \int_{0}^{\infty}(g(r)-1) \frac{\sin q r}{q r} 4 \pi r^{2} d r .
\end{aligned}
$$

On a introduit la fonction de distribution de paires $g(r)$, centrale en physique des liquides, qui représente justement la probabilité de trouver 2 particules séparées par la distance $r$. La densité à la distance $r$ d'une particule vaut $\rho g(r)$ où $\rho=N / V$ est la densité colloïdale en nombre. $S(q)$ et $g(r)$ sont donc reliés par une TF tout ce qu'il y a de plus simple. Le graal d'un physicien des liquides est de connaître $g(r)$. C'est donc aussi le but d'un expérimentateur qui veut pouvoir en déduire facteur de structure et intensité diffusée. A quoi peuvent donc ressembler ces différentes fonctions? La figure 2 donne quelques exemples de sphères dures (type billes de billard) à différentes fractions volumiques. Dans le cas idéal (sans aucune interaction, même de volume exclu), $g=1$ et $S=1$. Dans le cas très dilué "gaz", on verra plus bas l'expression asymptotique de $g$ mais, de toute façon, $S=1$ puisque $\rho=0$. Dans le cas complètement
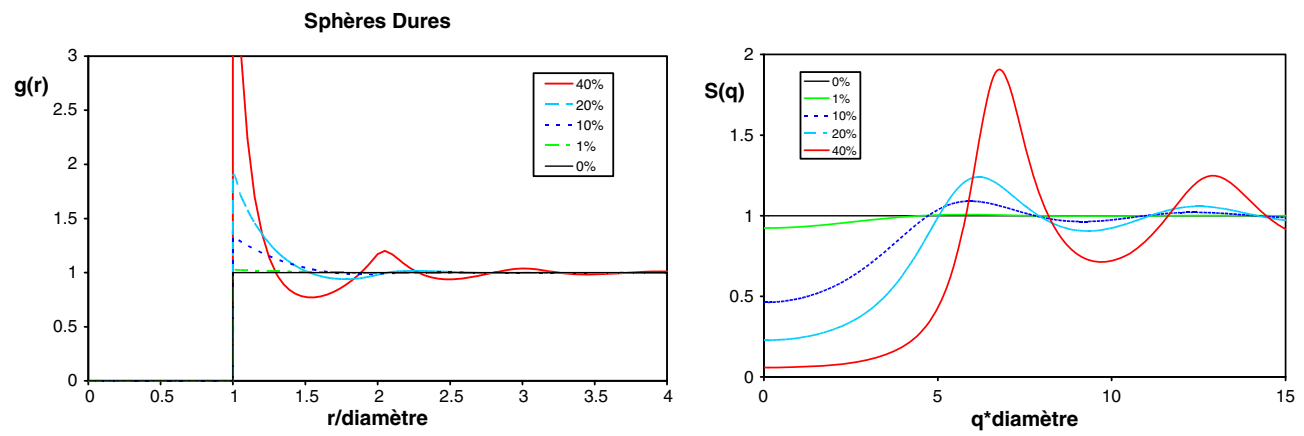

Figure 2. Fonction de distribution $g(r)$ et facteur de structure $S(q)$ pour un système de sphères dures pures à différentes fractions volumiques $(0-40 \%)$. 
opposé d'un cristal où les particules sont réparties régulièrement au centre ou au voisinage du centre de mailles cristallines, $g(r)$ présente une succession régulière de pics très fins (infiniment fins si les particules sont rigoureusement gelées au centre de leurs mailles) et $S(q)$ présente des fonctions de Dirac (si cristal infini) qui ne sont que les pics de Bragg du cristal en diffraction. Dans le cas intermédiaire d'un ordre liquide, on observe très bien dans la figure 2 un ordre à courte distance et un désordre au-delà. $g(r)$ et $S(q)$ tendent vers 1 à grand $r$ ou $q$. Les pics et oscillations amplifiés à concentration importante traduisent l'arrangement local des collö̈des les uns autour des autres. Une relation fondamentale, dite de la compressibilité, relie $S(0)$ à la compressibilité isotherme réduite :

$$
S(0)=\chi=k T(\partial \rho / \partial P)_{T}
$$

$T$ est la température (en $\mathrm{K}$ ) et $k$ la constante de Boltzmann. Pour un liquide simple, $P$ représente la pression tout court. Pour une solution (soluté de densité $\rho$ dans un solvant), $P$ représente la pression osmotique de la solution, différence de pressions absolues entre solution et solvant pur séparés par une membrane semi-perméable au solvant, étant entendu dans ce cas que la dérivée thermodynamique s'effectue à potentiel chimique du solvant constant. Le facteur de structure $S(0)$ (soluté-soluté) donne donc la compressibilité osmotique. Pour être complet, le lecteur attentif (et tatillon!) aura peut-être noté que faire $q=0$ dans la définition de $S(q)$ (1 ${ }^{\text {ère }}$ expression dans (9)) donne simplement $S(0)=N$ ! De même, il aura tiqué devant le remplacement sournois de $g$ par $g-1$ dans la dernière égalité de (9). En fait, le facteur de structure d'un volume diffusant macroscopique de taille $L \approx 1 \mathrm{~cm}$ vaut effectivement $N$ à l'origine mais il chute très vite, sur quelques $q \approx L^{-1}$, comme le fait le facteur de forme de n'importe quel objet uniforme de taille comparable, donc sur une gamme de $q$ beaucoup plus petite que celle qui caractérise les corrélations inter-particules, $\approx R^{-1}$, et qui est accessible expérimentalement. Remplacer $g$ par $g-1$ revient à ignorer ce pic central très fin (infiniment fin dans le cas d'un volume infini) et à se focaliser sur la distribution discrète (et non continue homogène) des colloïdes dans le milieu. $S(q=0)$ dans (10) représente donc en fait $L^{-1} \ll q \ll R^{-1}$

Une fois obtenu le facteur de structure, on le multiplie par le facteur de forme de la sphère (5) et on obtient l'intensité. L'exemple typique de la figure 3 correspond à une assemblée de sphères répulsives (les détails de l'interaction importent peu ici). A grand $q$, là où $\mathrm{S}=1$, on retrouve les oscillations non perturbées du facteur de forme (tant mieux si c'est le seul intérêt de l'étude). A petit $q$, l'intensité est fortement réduite en raison de la faible compressibilité liée aux répulsions. La figure 3 illustre aussi le fait que la chute rapide du facteur de forme à grand $q$ et le bruit expérimental toujours présent tuent de l'information importante sur $S(q)$ sur une grande gamme de $q$ est qu'il est illusoire de pouvoir "mesurer" en pratique $g(r)$ en divisant $I(q)$ expérimental par $P(q)$ expérimental et en effectuant une TF numérique.
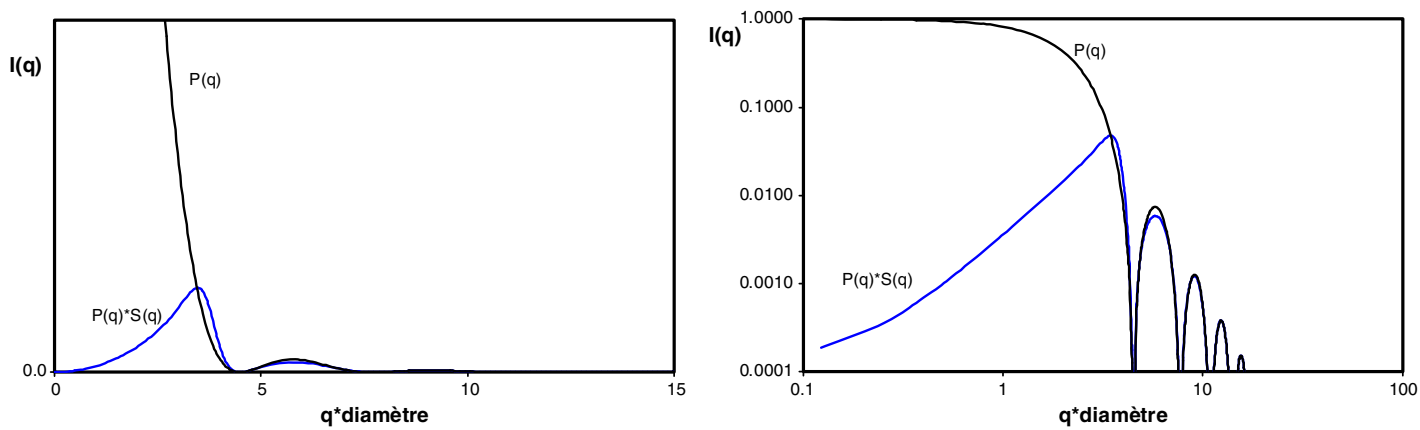

Figure 3. Intensité diffusée par une assemblée de sphères répulsives. Echelles lin-lin et log-log. 


\section{MÉCANIQUE STATISTIQUE DES LIQUIDES - LIEN ENTRE $v(r)$ ET $g(r)-S(q)$}

On sait donc maintenant exprimer simplement l'intensité diffusée en fonction du facteur de structure et de la fonction de distribution de paires. Comment relier ces deux grandeurs collectives au potentiel de paires microscopique $v(r)$ entre colloïdes, à température et concentration données? (on supposera dans la suite que les particules interagissent 2 à 2 ce qui est généralement le cas et on négligera les interactions à 3 corps ... ). C'est la question centrale de physique théorique des liquides. Pour les liquides simples, $v(r)$ représente l'interaction entre 2 atomes ou molécules dans le vide. Pour nos solutions colloïdales, à notre niveau de description mésoscopique qui ignore les détails du fluide porteur, $v(r)$ représente l'interaction effective entre 2 colloïdes, sous-entendue moyennée sur les degrés de liberté du solvant et des petits solutés. Elle présente donc une zoologie très riche et contrôlable, voir le chapitre dédié et des revues récentes [3]. On peut juste citer ici qu'elle peut être répulsive, $v>0$ (cœur dur ou mou, électrostatique, stérique, hydratation ...) ou attractive, $v<0$ (van der waals, dépletion, pontage ...) ou mixe (Lennard-Jones, DLVO). Pour bien illustrer le fait que le passage de $v$ à $g$ demande la résolution d'un problème compliqué à $N$ corps, voici l'expression formelle exacte dans l'ensemble canonique $(T$, $N$ et $V$ fixés) [4] :

$$
g\left(r_{12}\right)=\frac{V^{2} \int \exp \left[-\sum_{i<j} v\left(r_{i j}\right) / k T\right] d \vec{r}_{3} d \vec{r}_{4} \ldots d \vec{r}_{N}}{\int \exp \left[-\sum_{i<j} v\left(r_{i j}\right) / k T\right] d \vec{r}_{1} d \vec{r}_{2} d \vec{r}_{3} d \vec{r}_{4} \ldots d \vec{r}_{N}} .
$$

Comment éviter ces intégrales multiples et calculer rapidement et précisément $g(r)$ ? Trois types de "boîte noire" de mécanique statistique sont possibles. On se place toujours dans le cas de particules sphériques identiques.

\subsection{Développements du viriel}

Pendant longtemps, on s'est limité aux solutions diluées et on a exprimé les écarts à l'idéalité en puissances croissantes de la densité, en ne conservant généralement que le $1^{\text {er }}$ terme non trivial. Si l'on n'est pas capable de traiter $10^{23}$ particules en même temps, on devrait quand-même être capable d'en traiter deux! Tout est basé sur la limite asymptotique, évidente, de $g(r)$ à dilution infinie :

$$
g(r) \underset{\rho=0}{\longrightarrow} e^{-v(r) / k T}
$$

et donc

$$
S(0)=\chi \approx 1+\rho \int_{0}^{\infty}\left(e^{-v(r) / k T)}-1\right) 4 \pi r^{2} d r+\cdots .
$$

L'expression (13) est un exemple de développement du viriel, dont on ne conserve en général que le terme explicitement donné, le coefficient en $\rho$ étant alors appelé $2^{\text {nd }}$ coefficient du viriel $(-1 / 2$ fois l'intégrale pour être précis). Un développement similaire existe à $q$ fini mais, en pratique, on ne l'utilise que dans la limite $q=0$. Les tracés dits de Debye, de Zimm, extrapolés à concentration nulle, sont utilisés pour extraire le coefficient du viriel et obtenir des informations sur le potentiel. Je n'en dirai pas plus ici car cette analyse est limitée par essence au régime dilué (elle est donc sensible au bruit expérimental) et on peut faire beaucoup mieux pour à peine plus cher.

\subsection{Simulation numérique}

L'idée de base est que l'on peut reproduire le comportement d'un système macroscopique (taille $L \approx 1 \mathrm{~cm}, 10^{23}$ particules) en étudiant une "boîte" de simulation contenant seulement quelques centaines de particules mais dont la taille $L\left(N=\rho L^{3}\right.$ pour une boîte cubique) reste néanmoins grande devant les échelles caractéristiques microscopiques (comme le rayon $R$ ). Manipuler numériquement 
$N=10^{2}-10^{4}$ particules reste tout à fait raisonnable avec les ordinateurs actuels de bureau. On fabrique les trajectoires (des trajectoires) des particules dans la boîte à l'aide de déplacements Monte Carlo (MC) ou en résolvant pas à pas (de manière approchée) les équations de Newton (dynamique moléculaire) ou de Langevin (dynamique brownienne pour tenir compte du solvant visqueux sous-jacent) [5]. On utilise des conditions aux limites périodiques pour atténuer les effets de taille finie de la boîte. On effectue $10^{4}-10^{8}$ itérations pour bien explorer l'espace des phases, en tout cas ses régions les plus probables. On "prend des photos" régulièrement (voire des "films" si on s'intéresse aussi aux propriétés dynamiques), on compte les paires de particules séparées (environ) par la distance $r$ et on construit ainsi pas à pas $g(r)$.

L'intérêt premier de la simulation numérique est d'offrir un lien exact entre $v$ et $g$. Elle sert ainsi de référence contre laquelle vont se confronter des théories approchées plus rapides. Par contre, elle n'est pas vraiment adaptée au traitement de routine de spectres expérimentaux car chaque simulation, même simple, demande au moins plusieurs minutes de calcul (plusieurs semaines pour les calculs les plus lourds), n'apporte des informations que parcellaires (on ne connaît $g(r)$ que pour $r<L / 2$ ) et est toujours entachée d'un bruit statistique qui ne décroît que lentement avec le nombre de configurations explorées et la durée de la simulation (en $1 / \sqrt{ } / \mathrm{N}_{\text {config }}$ ).

\section{3 Équations intégrales}

C'est la boîte noire qui offre le meilleur compromis précision-rapidité et qui est recommandée pour l'analyse de spectres de diffusion. Le formalisme théorique a été développé dans les années 1955-1965 [4] et est utilisé en pratique pour les solutions colloïdales depuis les années 1980. Le point de départ est l'équation introduite phénoménologiquement par Ornstein et Zernike il y a beaucoup plus longtemps (1915) pour l'étude des phénomènes critiques et qui porte leur nom (OZ):

$$
h(r)=c(r)+\rho \int h\left(r^{\prime}\right) c\left(\left|\vec{r}-\vec{r}^{\prime}\right|\right) d \vec{r}^{\prime} .
$$

La fonction de corrélation totale $h=g-1$ est décomposée en une partie directe, la fonction de corrélation directe $c(r)$, et une partie indirecte qui s'exprime comme produit de convolution des deux (corrélation via une $3^{\text {ème }}, 4^{\text {ème }} \ldots$ particule). On peut considérer l'équation OZ comme la définition de $c$. Bien que moins fondamentale et intuitive que $g$, la physique des liquides moderne a montré comment cette fonction apparaît dans toute analyse théorique [4]. Ses propriétés à savoir ici est qu'elle se comporte comme $\exp (-v / k T)$-1 à dilution infinie et comme $-v / k T$ à grande séparation, quelle que soit la densité. La TF de (14) remplace le produit de convolution par un produit tout court et l'on obtient:

$$
S(q)=1+\hat{h}(q)=\frac{1}{1-\hat{c}(q)} .
$$

Les fonctions "chapeau" représentent des TF normalisées par la densité $\rho$ comme dans (9). OZ ont dit qu'au voisinage d'un point critique, la compressibilité et $S(0)$ divergeaient alors que $\hat{c}(0)$ tendait simplement vers 1.

On a donc une équation pour 2 inconnues, il nous en manque une seconde, dite relation de fermeture ou équation intégrale. Il existe une telle relation formelle exacte [4]:

$$
g(r)=\exp \left[-\frac{v(r)}{k T}+h(r)-c(r)+b(r)\right] .
$$

Le problème est que la fonction bridge $b(r)$ est une fonction(nelle) très compliquée des autres fonctions et n'est donc pas bien connue, voire inconnue. Tout juste peut-on dire ici que $b$ s'annule comme $\rho^{2}$ à grande dilution. Le but ultime dans cette approche de mécanique statistique des liquides est d'obtenir une bonne fonction bridge, aussi bonne que l'on peut. Différentes approximations pour $b$ existent dans la littérature, qui définissent différentes équations intégrales. La $1^{\text {ière }}$ consiste simplement à la négliger, 
$b=0$. Il s'agit de l'équation HNC :

$$
g(r)=\exp \left[-\frac{v(r)}{k T}+h(r)-c(r)\right] \text { HNC. }
$$

C'est une très bonne approximation pour la plupart des systèmes colloïdaux (je parle toujours au niveau à solvant continu implicite), en particulier pour les interactions électrostatiques. Si on linéarise par rapport à $h-c$, on obtient l'équation Percus-Yevick PY :

$$
g(r)=\exp \left[-\frac{v(r)}{k T}\right](1+h(r)-c(r)) \quad \mathrm{PY} .
$$

Là aussi, l'approximation est bonne (ce ne serait pas vrai pour des interactions électrostatiques non écrantées en $1 / r)$. HNC et PY donnent exactement les $2^{\text {ème }}$ et $3^{\text {ème }}$ coefficients du viriel. Si on linéarise l'ensemble de l'exponentielle dans (17), on obtient enfin l'équation MSA :

$$
c(r)=-v(r) / k T \quad \text { MSA. }
$$

On identifie ici la fonction directe à son comportement asymptotique longue distance. La linéarisation de $\exp (-v / k T)$ est évidemment catastrophique à forte interaction. On utilise donc MSA uniquement en présence d'un cœur dur de diamètre $\sigma=2 a$ (où on peut dire sans se tromper $g(r<\sigma)=0$ ) et au-delà de $\sigma$ seulement $(c(r>\sigma)=-v(r) / k T)$. Le gros intérêt (le seul?) de l'équation MSA ainsi définie est de donner lieu à une solution analytique (grâce à de la mathématique de haute volée!) pour quantité de potentiels: sphères dures (SD) pures (dans ce cas, MSA et PY coïncident), SD + Yukawa (potentiel en $\exp (-\alpha r) / r)$ ), SD chargées, SD + multipôles, mélanges de particules avec mélanges de ces potentiels ...En particulier, l'usage de la solution de SD + Yukawa [6] a été popularisé au début des années 1980 par Hayter et Penfold [7] et repris un nombre incalculable de fois depuis pour l'analyse des solutions de colloïdes chargés qui interagissent via le potentiel coulombien écranté DLVO (on néglige ici la contribution $\mathrm{VdW}$ ) [8] :

$$
\beta v(r)=\frac{Z_{e f f}^{2} L_{B}}{(1+\kappa a)^{2}} \exp (-\kappa r) / r
$$

$\beta=1 / k T, L_{B}=e^{2} /\left(4 \pi \varepsilon_{0} \varepsilon k T\right)$ est la longueur de Bjerrum, $\kappa$ est la constante d'écrantage qui dépend de la force ionique, $Z_{\text {eff }}$ est la charge effective, post condensation ionique, du colloïde. Mais attention quand on utilise MSA, il faut toujours garder à l'esprit que cette approximation est très mauvaise dès que le potentiel devient grand devant $k T$, ce qui arrive malheureusement pour la répulsion électrostatique près du contact. Sans précaution, on trouverait $g<0$ ! Hayter et Hansen [9] ont alors proposé une rustine qui marche bien et qui consiste à remplacer la répulsion très grande par une répulsion infinie, donc à augmenter, "renormaliser" le diamètre de cœur dur de $\sigma$ à $\sigma^{\text {eff }}$. Du point de vue physique, cela ne doit pas changer grand-chose puisque, de toute façon, les collö̈des sont très rarement près du contact. Du point de vue MSA, cela change tout car on dit $g\left(r<\sigma^{\text {eff }}\right)=0$. Jusqu'où augmenter $\sigma^{\text {eff }}$ ? Le critère proposé est $g\left(\sigma^{\text {eff }}\right)=0$. Cela définit l'équation RMSA [9] qu'il faut absolument utiliser à la place de MSA dans ce cas.

Pour l'équation PY, une autre solution analytique importante est disponible, celle pour le potentiel adhésif [10]. Il s'agit d'une attraction au contact de profondeur "infinie" et de portée "nulle", mathématiquement traduite par $\exp (-v(r) / k T)=v \delta(r-\sigma)$. C'est un bon modèle pour une attraction physique réelle à courte portée (l'adhésivité $v$ étant alors reliée à exp(profondeur/ $k T$ )*largeur) ou pour une attraction chimique (l'adhésivité $v$ étant proportionnelle à la constante d'association dans la loi d'action de masse).

En dehors de ces cas isolés, l'équation intégrale requiert une résolution numérique qui, du coup, peut être utilisée pour n'importe quelle forme de potentiel, aussi complexe soit-elle. 


\subsection{Résolution numérique itérative}

Les 2 équations, OZ et fermeture, s'expriment simplement l'une dans l'espace de Fourier, l'autre dans l'espace réel. La résolution numérique sera donc itérative et fera plein d'aller-retour entre les 2 représentations via des TF numériques. Une itération correspond au parcours du cycle schématique suivant :

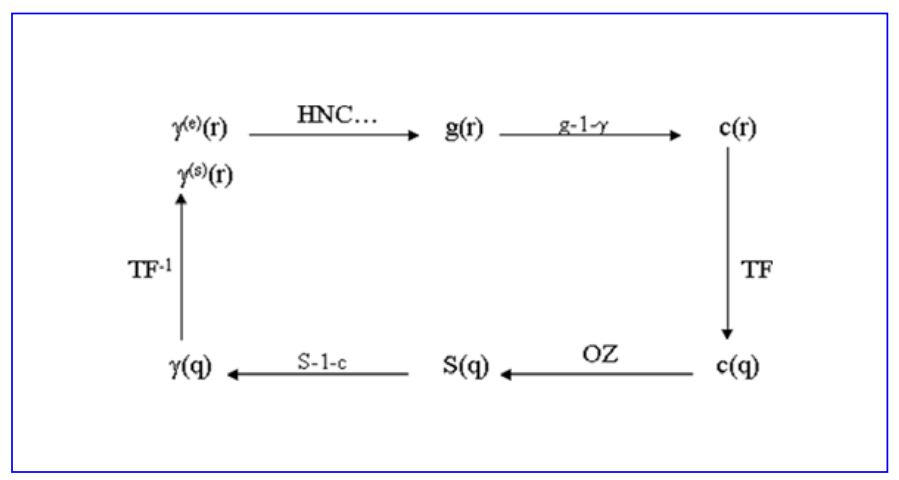

Figure 4. Résolution itérative d'une équation intégrale.

On part d'une fonction $\gamma(r)=h(r)-c(r)$ d'entrée, $\gamma^{(\mathrm{e})}$, on décrit le cycle via fermeture, TF, OZ, $\mathrm{TF}^{-1}$, et on arrive à la fonction de sortie $\gamma^{(\mathrm{s})}$. Et on recommence jusqu'à ce que ces 2 fonctions coïncident à une tolérance près (disons $10^{-4}$ ). On démarre le cycle suivant de manière frustre (mélange de fonctions d'entrée et de sortie du cycle actuel) ou de manière futée (technique Newton-Raphson de course) [11]. Chaque fonction est discrétisée sur 512-2048 points, les TF sont réalisées par FFT. Une résolution pour un système colloïdal standard est obtenu en quelques secondes (je répète, pour des sphères et au niveau solvant implicite).

Les différentes boîtes noires sont comparées en figure 5 pour un système classique de colloïdes nanométriques. La simulation MC sert de référence. Le pic à angle nul avec les oscillations à ses pieds que l'on devine à petit $q(<1 / L)$ n'est autre que la signature de la taille finie $L$ de la boîte de simulation et illustre les limites pratiques de cette technique. Il faudrait une boîte beaucoup plus grande pour pouvoir extraire convenablement $S($ “"0”). L'accord MC-HNC en $g(r)$ et en $S(q>1 / L)$ est très bon. Au contraire, les courbes MSA sont, comme attendu, complètement fausses ( $v$ vaut $11 k T$ au contact!).
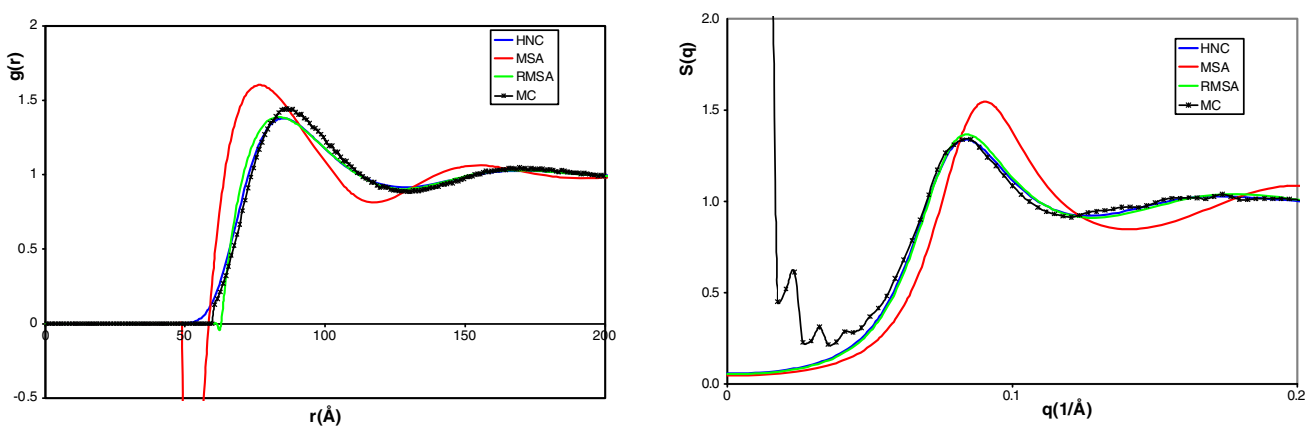

Figure 5. $g(r)$ et $S(q)$ pour une solution de colloïdes, potentiel (20), diamètre $50 \AA$, charge effective 20 , concentration $2.510^{-3} \mathrm{M}$ (fraction volumique 10\%) sans sel ajouté (seuls les contreions libres participent à la force ionique). 

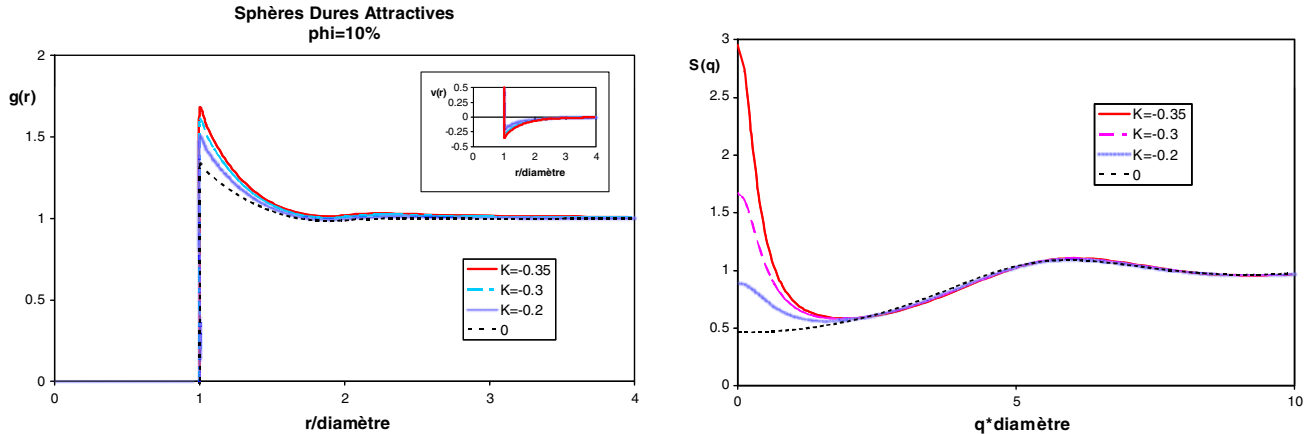

Figure 6. $g(r)$ et $S(q)$ pour une solution de sphères dures à $10 \%$ avec attraction supplémentaire croissante. Le potentiel attractif est tracé en insert, $K$ est la profondeur au contact (en $k T$ ).

RMSA renormalise alors à $\sigma^{\text {eff }}=63 \AA$ et la courbe $S(q)$ devient très correcte. Du point de vue physique, les pics principaux observés en $r$ et en $q$ traduisent l'arrangement (liquide) 3D des colloïdes au sein de la solution du fait de leur répulsion longue portée. Leur position ne dépend que de la concentration, $r \approx \rho^{-1 / 3}$. C'est à opposer au cas d'une répulsion courte portée comme SD pure (figure 2) où le pic de $g(r)$ est au contact, $r=\sigma$. La comparaison avec les données expérimentales se fait alors au niveau $I(q)$ après multiplication par le facteur de forme. Si l'accord n'est pas suffisant, on recommence en changeant certains paramètres du potentiel (taille, charge effective ...) ou en ajoutant de nouvelles contributions au potentiel.

Les figures 2,5 ont montré des exemples de répulsion. La figure 6 présente le cas de sphères dures avec potentiel attractif (par exemple déplétion due à la présence de petits polymères non adsorbants dans la solution) dont on augmente très doucement la profondeur au contact (la concentration de polymère). Contrairement à précédemment, une interaction modeste (inférieure à $k T$ ) peut avoir de gros effets (le facteur de Boltzmann est beaucoup plus sensible aux attractions qu'aux répulsions). Ces effets se voient à $q=0$ où un pic pousse. La solution devient de plus en plus compressible (osmotiquement) et l'on s'approche d'une transition de phases de type liquide-gaz (solution concentrée-solution diluée).

On peut aussi rencontrer des cas plus bizarres où attraction à courte portée et répulsion à plus longue portée cohabitent. L'agrégation locale due à la première est inhibée par la seconde et le résultat est la formation de clusters de taille bien définie qui se remarquent par la présence d'un pré-pic à $q$ fini dans le facteur de structure [12].

\section{MÉLANGE DE SPHÈRES}

On peut avoir à traiter des mélanges dans différentes situations: cela peut être une polydispersité résiduelle subie, qui résulte d'une synthèse pas optimale. On peut au contraire mélanger volontairement deux types de colloïde, jouer sur le contraste de chacun d'eux en DNPA et observer comment les petits s'organisent autour des gros. Enfin, on peut être beaucoup plus ambitieux, vouloir quitter le niveau de description colloïdal pour un niveau plus détaillé, et tenir compte explicitement par exemple des petits ions ou des petits polymères (mais solvant aqueux toujours continu) qui sont à la source de cette interaction colloïdale. Le formalisme de la section 3 est facilement généralisable. Si l'on a $n$ composants (toujours sphériques), on a donc $n$ facteurs d'amplitude $f_{\alpha}(\mathrm{q})$ et de forme $P_{\alpha}(\mathrm{q})$ et $n(n+1) / 2$ fonctions de distribution $g_{\alpha \beta}(r)$ et facteurs de structures partiels $S_{\alpha \beta}(q)$. L'intensité diffusée s'écrit comme combinaison linéaire des interférences $\alpha \beta$ :

$$
I(q)=\sum_{\alpha, \beta=\text { composants }} f_{\alpha} f_{\beta}\left\langle\sum_{i \in \alpha, j \in \beta} e^{i \vec{q} \vec{q}_{i j}}\right\rangle=\sum_{\alpha, \beta} \sqrt{N_{\alpha} N_{\beta}} f_{\alpha} f_{\beta} S_{\alpha \beta}
$$




$$
S_{\alpha \beta}(q)=\delta_{\alpha \beta}+\sqrt{\rho_{\alpha} \rho_{\beta}} \int_{0}^{\infty}\left(g_{\alpha \beta}(r)-1\right) \frac{\sin q r}{q r} 4 \pi r^{2} d r .
$$

L'équation intégrale HNC pour chaque paire $\alpha \beta$ s'écrira :

$$
g_{\alpha \beta}(r)=\exp \left[-\frac{v_{\alpha \beta}(r)}{k T}+\gamma_{\alpha \beta}(r)\right]
$$

alors que l'équation $\mathrm{OZ}$ prendra une forme matricielle dans l'espace de Fourier :

$$
S(q)=(I-C(q))^{-1} .
$$

La matrice $C$ est constituée de la TF des fonctions $c_{\alpha \beta}$, normalisées comme dans (22). La résolution numérique est similaire à la précédente, la seule nouveauté technique étant dans l'inversion de matrice (24) à chaque itération. Quelques secondes suffisent là-aussi pour la résolution complète.

\subsection{Polydispersité intempestive subie}

Un problème archi classique est le suivant: on étudie une solution a priori monodispersée. On commence par utiliser la formule à un composant $(8) P(q) S(q)$ mais on constate que les oscillations expérimentales à grand $q$ sont gommées, signe sans doute qu'il y a une certaine distribution de taille colloïdale mais donc aussi une distribution d'interaction colloïdale. On voudrait tenir compte (facilement comme dans (6)) de la $1^{\text {ère }}$ sans avoir à se coltiner la $2^{\text {nde }}$. De combien se trompe-t-on? Ne tenir compte de la polydispersité que dans le pouvoir diffusant des particules revient à utiliser une fonction $g(r)$ commune pour tous les couples $\alpha \beta$. L'intensité (21) devient alors :

$$
\begin{aligned}
& I(q)=N_{\text {tot }}\left[\left\langle f^{2}\right\rangle+\langle f\rangle^{2}(S-1)\right]=N_{\text {tot }}\left[\left\langle f^{2}\right\rangle-\langle f\rangle^{2}+\langle f\rangle^{2} S\right] \\
& \langle f\rangle=\frac{1}{N_{\text {tot }}} \sum_{\alpha} N_{\alpha} f_{\alpha} ; \quad\left\langle f^{2}\right\rangle=\frac{1}{N_{\text {tot }}} \sum_{\alpha} N_{\alpha} f_{\alpha}^{2} .
\end{aligned}
$$

Il n'y a plus qu'un seul $S(q)$ (9) (où $\rho$ représente la densité totale), c'est le but du jeu, que l'on calcule pour un système à un composant à partir d'une interaction commune moyenne. La figure 7 présente le cas de la figure 5 avec une polydispersité ternaire en taille. La polydispersité lisse les oscillations à grand $q$ comme attendu car $P$ est remplacé par le facteur de forme moyen $\left\langle f^{2}\right\rangle$, et ajoute du signal "incohérent" $\left\langle f^{2}\right\rangle-\langle f\rangle^{2}$ à petit $q$ (là où $S$ est d'habitude petit). Sur la même figure apparaît également la courbe correcte obtenue en tenant compte du mélange de potentiels, en ayant calculé les 6 facteurs
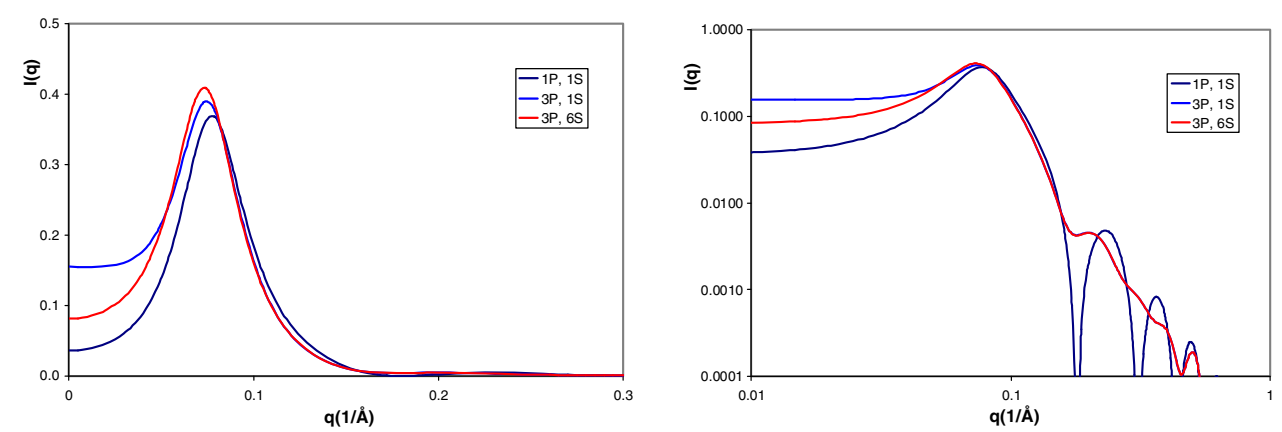

Figure 7. Intensité diffusée pour un mélange de 3 populations de sphères dures chargées interagissant via des potentiels coulombiens écrantés (20): diamètres $=40,50,60 \AA$, charges effectives $=16,20,24$, proportions en nombre $1,2,1$, fraction volumique totale $10 \%$. " $1 \mathrm{P}, 1 \mathrm{~S}$ " correspond à l'expression (8), "3P, $1 \mathrm{~S}$ " à (25), "3P, $6 \mathrm{~S}$ " à (21). 

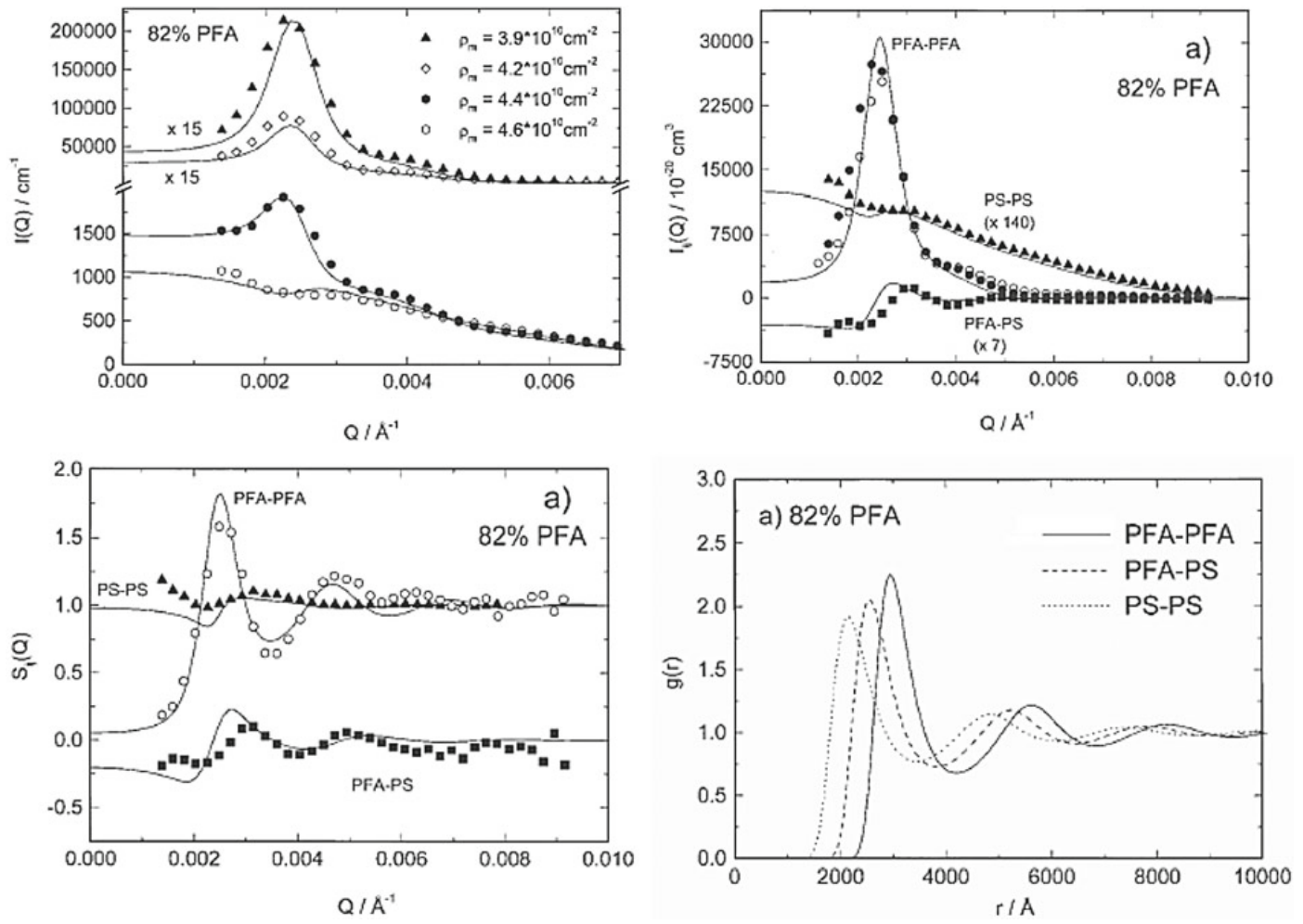

Figure 8. Mélange binaire de PS et PFA. En haut à gauche, intensité diffusée à différentes compositions du solvant $\mathrm{H}_{2} \mathrm{O} / \mathrm{D}_{2} \mathrm{O}$. En haut à droite, les 3 intensités partielles extraites. En bas à gauche, les 3 facteurs de structure partiels. Les points correspondent à la DNPA (ILL), les courbes à l'équation intégrale HNC qui donne les 3 fonctions de distribution en bas à droite [13].

de structure partiels (ici par HNC) et en ayant utilisé (21) pour l'intensité. On peut enfin répondre à la question initiale: ne tenir compte de la polydispersité que dans la forme et pas dans la structure va dans le bon sens mais a tendance à surestimer l'effet. L'explication physique est qu'on néglige l'arrangement spontané des petits autour des gros qui relâche les contraintes dues au mélange. Il faut donc réserver la formule simplifiée (25) au cas de polydispersités modestes.

\subsection{Mélange binaire de particules chargées polystyrène (PS) et perfluorinées (PFA)}

Cette étude [13] est un bel exemple du mariage de la DNPA et des équations intégrales dans le cas d'un mélange voulu. Brièvement, les 2 particules ont des densités de longueur de diffusion très différentes (elles ont été synthétisées pour ça). La méthode de variation de contraste avec solvants $\mathrm{H}_{2} \mathrm{O} / \mathrm{D}_{2} \mathrm{O}$ de différentes compositions permet de remonter aux 3 contributions $\alpha \beta$ à l'intensité (21) puis aux 3 facteurs de structure partiels après division par les facteurs d'amplitude. La résolution de l'équation HNC pour des potentiels DLVO (20) permet de reproduire avec beaucoup de détails ces différents termes. La figure (8) illustre un tel traitement pour un mélange $82 \%$ en PFA. Les détails sont à lire dans les articles originaux [13].

\subsection{Mélange colloïdes chargés-ions}

D'où vient le potentiel coulombien écranté DLVO (20)? Quelle est sa validité? Pour connaître précisément l'interaction colloïdale entre colloïdes chargés, sous-entendue moyennée sur les ions, il 

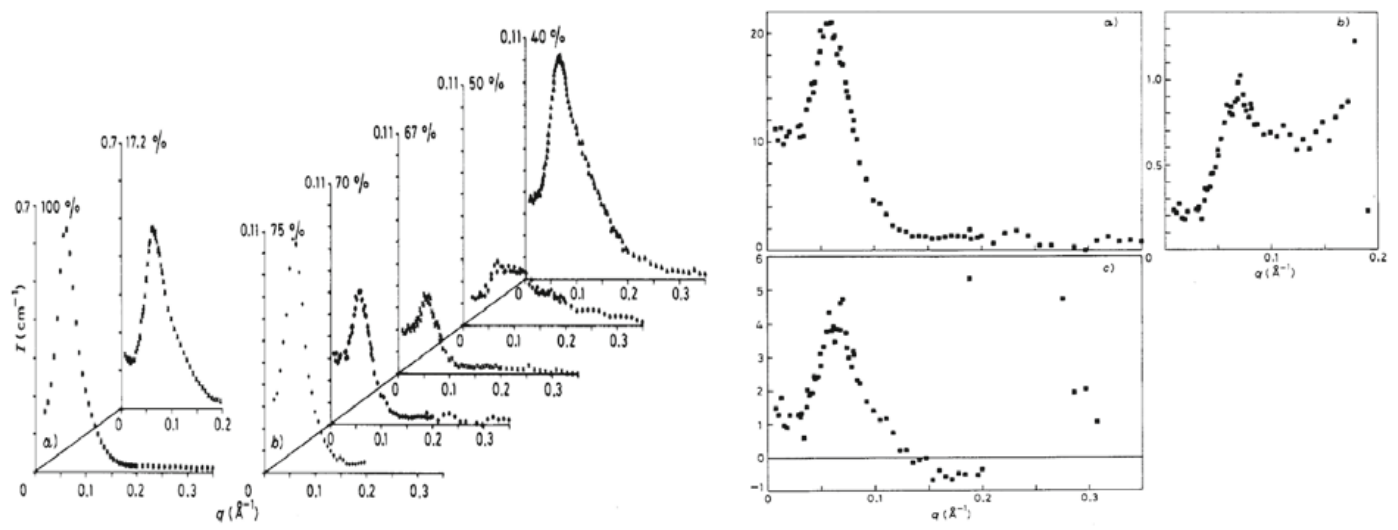

Figure 9. Solution micellaire de TMADS. A gauche, spectres DNPA (Orphée PACE) à différentes compositions $\mathrm{H}_{2} \mathrm{O} / \mathrm{D}_{2} \mathrm{O}$. A droite, les 3 facteurs de structure partiels extraits, micelle-micelle, micelle-contreion et contreioncontreion [16].

faut se placer à un niveau de description plus fin et traiter les petits ions explicitement, sur le même pied d'égalité que les colloïdes. Le Modèle Primitif considère ainsi des mélanges de sphères plongées dans un solvant diélectrique (toujours continu, c'est en ça que le modèle est primitif) et interagissant via les potentiels coulombiens $q_{i} q_{j} / 4 \pi \varepsilon_{0} \varepsilon r$ où $\varepsilon$ est la constante diélectrique du solvant caché. Le problème de mécanique statistique est nettement plus délicat que précédemment: on a affaire à des mélanges très dissymétriques en taille, charge et nombre, les potentiels coulombiens, même divisés par $\varepsilon$, sont généralement nettement plus grands que $k T$, ils sont de portée "infinie", les répulsions ++ et - côtoient les attractions $+-\ldots$ En conséquence, les simulations numériques sont longues et limitées aux charges colloïdales modestes, l'équation intégrale HNC, toujours résolue en quelques secondes [14], devient moins valable qu'au niveau de description colloïdal et toute une littérature cherche à l'améliorer depuis 25 ans à l'aide de fonctions bridges approchées ... Pour toutes ces raisons, le Modèle Primitif n'est pas adapté au traitement de routine de spectres expérimentaux et est surtout utilisé au niveau théorique pour élucider la condensation électrostatique des contreions au voisinage des surfaces colloïdales et pour montrer comment l'interaction colloïdale, cohérente avec DLVO (20) pour des ions monovalents, peut devenir attractive en présence de contreions multivalents [15]! Néanmoins, il existe un cas où le traitement explicite des ions est requis pour l'analyse de spectres, c'est quand les ions participent eux-mêmes à la diffusion. La figure 9 donne un exemple de DNPA. Il s'agit de solutions micellaires de TMADS (on part de solutions classiques de SDS et on remplace le contreion sodium par le contreion tétraméthylammonium hydrogéné à fort pouvoir diffusant) et on mesure l'intensité diffusée à différents contrastes (mélanges $\mathrm{H}_{2} \mathrm{O} / \mathrm{D}_{2} \mathrm{O}$ ) pour extraire les 3 facteurs de structure partiels (Orphée, PACE) [16].

\section{PARTICULES RIGIDES ANISOTROPES}

On est de plus en plus ambitieux, on veut maintenant traiter le cas de particules non sphériques dont les facteurs d'amplitude instantanés dépendent de leurs orientations individuelles (par rapport au vecteur $q$ ) et dont leurs corrélations de paires dépendent de leurs orientations relatives. L'expression de départ (2) peut s'écrire formellement :

$$
I(q) / N=\int|f(\Omega)|^{2} d \Omega+\iint\left[f\left(\Omega_{1}\right) f^{*}\left(\Omega_{2}\right) \rho \int h\left(\vec{R}, \Omega_{1} \Omega_{2}\right) e^{i \vec{q} \vec{R}} d \vec{R}\right] d \Omega_{1} d \Omega_{2} .
$$

La fonction de distribution de paires dépend cette fois-ci non seulement de la distance entre les origines des 2 particules mais aussi des angles solides relatifs des 2 particules et du vecteur les joignant 


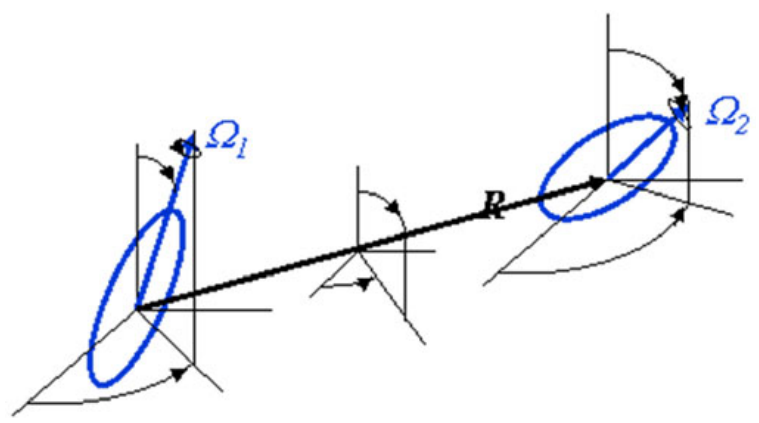

Figure 10. Particules anisotropes. Une configuration d'orientation est repérée par 5 angles d'Euler (3 pour particules linéaires).

(figure 10). Pour des particules linéaires, à axe de symétrie, comme les ellipsoïdes de révolution, les cylindres, les dipôles ..., 3 angles d'Euler suffisent pour repérer une configuration d'orientation, par exemple les 2 co-latitudes et la différence de longitude dans le repère intermoléculaire lié au vecteur $R_{12}$. Pour des particules non linéaires comme les molécules $\mathrm{H}_{2} \mathrm{O}$, les "molécules colloïdales" (assemblée de colloïdes sphériques vus comme des atomes) ..., il faut 5 angles d'Euler (rajouter la rotation des 2 particules autour de leur axe principal). Comme on peut l'imaginer, le traitement théorique peut devenir rapidement prohibitif !

\subsection{Anisotropie en forme seulement, pas en structure}

Comme pour le cas 4.1, on peut vouloir faire simple et n'envisager d'introduire l'anisotropie que dans le facteur d'amplitude individuel des particules en la négligeant dans les corrélations de paires. On considère donc que $g(r)$ ne dépend que de $r$, le facteur de structure $S(q)$ est calculé à partir d'un modèle de sphères qui représente grossièrement les particules, et l'intensité (26) devient:

$$
\begin{aligned}
& I(q)=N\left[\left\langle f^{2}\right\rangle+\langle f\rangle^{2}(S-1)\right]=N\left[\left\langle f^{2}\right\rangle-\langle f\rangle^{2}+\langle f\rangle^{2} S\right] \\
& \langle f\rangle=\int f(\Omega) d \Omega / 4 \pi ; \quad\left\langle f^{2}\right\rangle=\int|f(\Omega)|^{2} d \Omega / 4 \pi .
\end{aligned}
$$

Cette expression ressemble formellement beaucoup à celle vue plus haut (25), les moyennes sur les types de sphère étant remplacées par des moyennes sur l'orientation individuelle $\Omega$ de la particule autour de $q$ (1 ou 2 angle(s)). Dans les 2 cas, les oscillations à grand $q$ sont lissées, ce qui est en général le but recherché. Une différence importante: contrairement à 4.1, il n’y a plus ici de signal rajouté à petit $q$, l'anisotropie de forme étant indétectable à $q=0$.

Question traditionnelle: de combien se trompe-t-on quand on néglige ainsi l'anisotropie des corrélations? La figure 11 illustre le cas de particules en forme d'haltères. La courbe de référence (26) a été obtenue en traitant explicitement les interactions et donc corrélations anisotropes à l'aide de l'équation intégrale HNC. Les dimères interagissent via 4 interactions site-site sphériques LennardJones. L'expression simpliste (8), $P(q) S(q)$, obtenue avec $P$ et $S$ de sphères "équivalentes", manque à la fois les oscillations de forme à grand $q$ et l'ordre à petit $q$. La formule intermédiaire (27) reproduit bien-sûr correctement le facteur de forme des haltères à grand $q$ mais est toujours déficiente dans la reproduction de l'ordre local (bien que l'on ait choisi les sphères équivalentes de façon à reproduire le même $I(0)$ ). Ce cas particulier illustre les mérites et limites de l'expression (27). Quand on ne peut pas faire mieux que des corrélations sphériques, on est forcément obligé de s'en contenter.... 


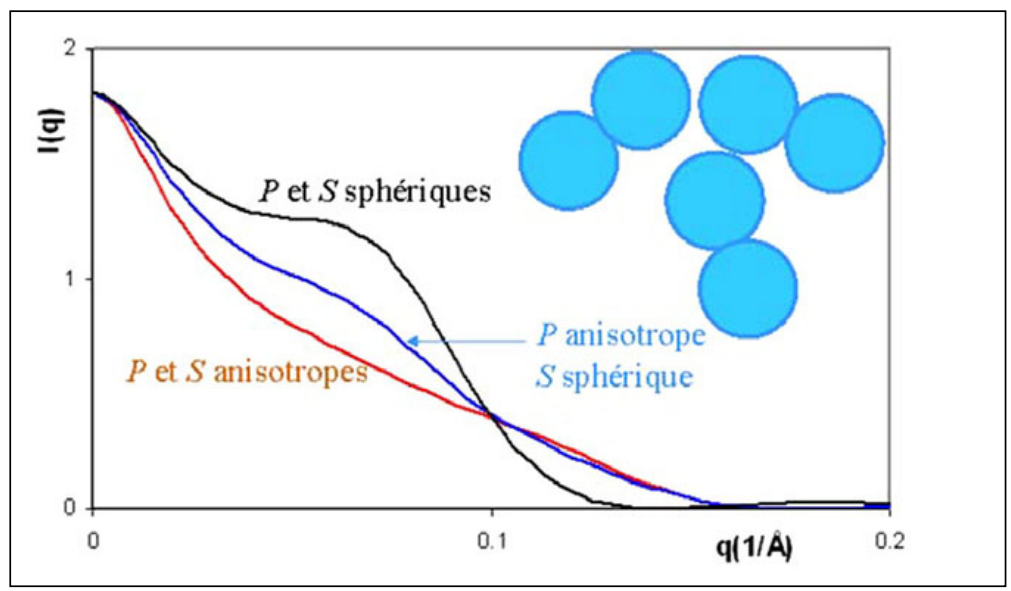

Figure 11. Solutions de dimères "haltères" constitués de 2 sites LJ à fraction volumique $20 \%$.

\subsection{Corrélations anisotropes}

On s'écarte un peu du sujet initial, traiter des données de diffusion de manière simple et systématique, et ce n'est pas le lieu pour détailler la mécanique statistique de particules non sphériques. Je voudrais juste dire que beaucoup de simulations numériques et de résolutions d'équations intégrales de type HNC ont été réalisées depuis de nombreuses années pour l'étude d'électrolytes au niveau de description à solvant discret. Il s'agit cette fois-ci de considérer un mélange de molécules $\mathrm{H}_{2} \mathrm{O}$, d'ions et, éventuellement, de colloïdes ou interfaces, qui interagissent via des potentiels électrostatiques ou multipolaires dans le vide, en $1 / r$. Les couplages sont très forts, bien plus forts qu'au niveau à solvant continu, les corrélations sont très directionnelles comme dans la structure tétraédrique de l'eau ou dans les couches d'hydratation. Des programmes numériques performants permettent de résoudre l'équation intégrale $\mathrm{HNC}$ en quelques minutes. Malgré son caractère approché, cette équation reproduit raisonnablement bien ce qui se passe au sein de la solution ou au voisinage d'interfaces. Les corrélations ion-ion obtenues sont interprétées en termes de potentiels effectifs ion-ion, c'est-à-dire moyennés sur le solvant. Au comportement asymptotique attendu en $1 / \varepsilon r$ ( $\varepsilon$ est ici un résultat du modèle) se surimpose une contribution à courte portée organisée, manquante dans le Modèle Primitif de base, qui traduit la structure discrète des molécules d'eau autour des ions. Ce qui est important pour nous ici, c'est que ces mêmes programmes commencent à être utilisés pour étudier (au niveau à solvant+ions implicites) les corrélations de particules colloïdales anisotropes et à traiter des données de diffusion de rayonnement, la figure 11 en est un exemple. On peut espérer que cette méthode d'analyse va se "démocratiser" dans le futur proche.

\section{ORGANISATIONS 1D ET 2D}

On a discuté jusqu'à présent de solutions de particules qui s'organisent en 3 dimensions. Sans prétention d'exhaustivité, il faut signaler qu'il est possible également de faire appel à la mécanique statistique dans le cas d'organisations à $1 \mathrm{D}$ ou $2 \mathrm{D}$, toujours observées en diffusion aux petits angles, en particulier en DNPA.

Un bel exemple d'organisation 1D est le cas des phases smectiques-A. Il s'agit par exemple de phases lamellaires constituées d'un sandwich de bicouches chargées plongées dans l'eau. Si les bicouches sont déposées sur un substrat plan macroscopique, la réflectivité est la technique expérimentale de choix et donne les corrélations perpendiculaires (spéculaires) et parallèles (hors spéculaires) aux plans. S'il 


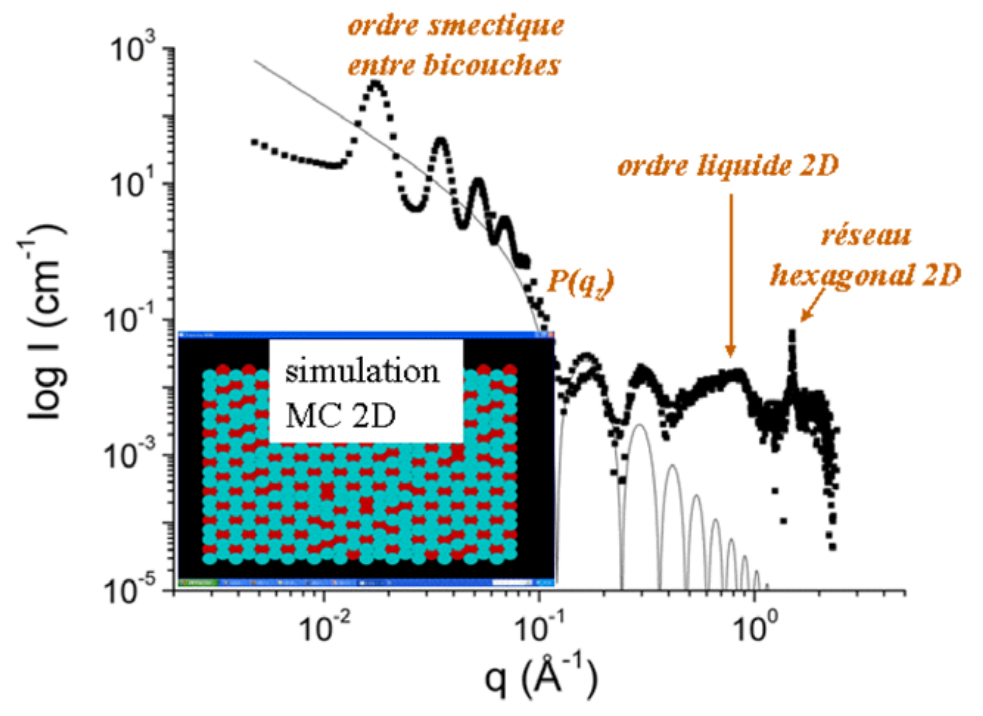

Figure 12. Diffusion de neutrons aux petits et grands angles (ILL D16 et D22) pour un système lamellaire de bicouches catanioniques. La courbe continue présente le facteur de forme des bicouches. En insert, configuration typique Monte Carlo de l'ordre liquide des tensioactifs + et - au sein du réseau hexagonal des bicouches [17].

s'agit de vésicules multi-lamellaires, la diffusion aux petits angles donne la moyenne de poudre des précédentes. Dans tous les cas, l'intensité diffusée est reliée au facteur de forme (perpendiculaire) des membranes, à leurs propriétés d'élasticité et aux interactions entre bicouches voisines via l'eau et les ions. Le traitement théorique n'est pas si loin de celui présenté jusqu'à maintenant, il s'agit toujours de manipuler des facteurs de Boltzmann.

Je terminerai avec l'exemple très riche des systèmes catanioniques, mélanges de tensioactifs cationiques et anioniques, qui forment spontanément sous certaines conditions des systèmes lamellaires de bicouches très rigides. Les 2 types de tensioactif, de pouvoirs diffusants différents, occupent au sein de chaque monocouche les mailles d'un réseau hexagonal [17]. Comme l'indique la figure 12, un même spectre de DNPA (jusqu'à $2 \AA^{-1}$ ) est capable de voir (en moyenne de poudre) à la fois l'ordre smectique (1D) entre bicouches à petit $q$, suivi du facteur de forme (perpendiculaire) des bicouches, le pic de Bragg du réseau hexagonal 2D à grand $q$ et, entre les 2 , à $q$ intermédiaire, le pic "mou" de l'ordre liquide 2D entre tensioactifs + et - qui résulte des interactions électrostatiques au sein du même réseau! La figure 12 montre également une configuration typique obtenue par simulation Monte Carlo (les tensioactifs préfèrent être entourés par des voisins de signe opposé) qui reproduit correctement ce pic liquide 2D (voir la référence originale [17] pour la comparaison DNPA-Monte-Carlo).

\section{Références}

[1] P. Maillet, C. Levard, E. Larquet, C. Mariet, O. Spalla, N. Menguy, A. Masion, E. Doelsch, J. Rose et A. Thill, J. Am. Chem. Soc. 132,1208 (2010)

[2] D.I. Svergun et K.H. Nierhaus, J. Biol. Chem. 275, 14432 (2000)

[3] L. Belloni, J. Phys. Cond. Matter 12, R549 (2000)

[4] J.P. Hansen et I.R. McDonald, Theory of Simple Liquids, London Academic (1986)

[5] M.P. Allen et D.J. Tildesley, Comuter Simulation of Liquids, Oxford Science (1987)

[6] E. Waisman, Mol. Phys. 25, 45 (1973)

[7] J.B. Hayter et J. Penfold, J. Chem. Soc. Faraday trans. I 77, 1851 (1981) 
[8] E.J.W. Verwey et J.Th.G. Overbeek, Theory of Stability of Lyophobic Colloids, Elsevier Amsterdam (1948)

[9] J.P. Hansen et J.B. Hayter, Mol. Phys. 46, 651 (1982)

[10] R.J. Baxter, J. Chem. Phys. 49, 2770 (1968)

[11] L. Belloni, J. Chem. Phys. 88, 5143 (1988)

[12] V. Peyre, O. Spalla, L. Belloni et M. Nabavi, J. Col. Interf. Sci. 187, 184 (1997)

[13] N. Lutterbach, H. Versmold, V. Reus, L. Belloni et Th. Zemb, Langmuir 15, 337 et 345 (1999)

[14] L. Belloni, Chem. Phys. 99, 43 (1985)

[15] L. Guldbrand, B. Jönsson, H. Wennerström et P. Linse, J. Chem. Phys. 80, 2221 (1984)

[16] P.J. Dérian, L. Belloni et M. Drifford, Europhys. Lett. 7, 243 (1988)

[17] D. Carrière, L. Belloni, B. Demé, M. Dubois, C. Vautrin, A. Meister et Th. Zemb, Soft Matter 5, 4983 (2009) 